\title{
Demographics of Head and Neck Cancer Patients: A Single Institution Experience
}

George S. Stoyanov ${ }^{1}$, Martina Kitanova ${ }^{2}$, Deyan L. Dzhenkov ${ }^{1}$, Peter Ghenev ${ }^{3}$, Nikolay Sapundzhiev ${ }^{4}$

1. General and Clinical Pathology, Forensic Medicine and Deontology, Medical University, Varna, BGR 2. Department of General and Clinical Pathology, Forensic Medicine and Deontology, Faculty of Medicine, Medical University - Varna “Prof. Dr. Paraskev Stoyanov”, Varna, Bulgaria 3. General and Clinical Pathology, Forensic Medicine and Deontology, Faculty of Medicine, Varna, BGR 4. Department of Neurosurgery and Ent Diseases, Division of Ent Diseases, Faculty of Medicine, Medical University - Varna "Prof. Dr. Paraskev Stoyanov", Varna, Bulgaria

$\square$ Corresponding author: George S. Stoyanov, georgi.geesh@gmail.com Disclosures can be found in Additional Information at the end of the article

\section{Abstract}

\section{Introduction}

Head and neck cancer (HNC) comprises a diverse group of oncological entities, originating from various tissue types and organ localizations, situated in the topographical regions of the head and neck $(\mathrm{H} \& N)$. This single institution retrospective study was aimed at establishing the HNC patient demographics and categorizing the individual incidence of $\mathrm{H} \& \mathrm{~N}$ malignancies, regarding their organ of origin and main histopathological type.

\section{Materials and methods}

All histologically verified cases of HNC from a single tertiary referral center were reviewed in a descriptive retrospective manner. Data sampling period was 47 months.

\section{Results}

Male to female ratio of the registered HNC cases was 3.24:1. The mean age of diagnosis was $63.84 \pm 12.65$ years, median 65 years. The most common HNC locations include the larynx $30.37 \%(n=188)$, lips and oral cavity $29.08 \%(n=180)$, pharynx $20.03 \%(n=124)$ and salivary glands $10.94 \%(n=68)$, with other locations such as the external nose, nasal cavity and sinuses and auricle and external ear canal harboring a minority of the cases. The main histopathological groups include squamous cell carcinoma 76.74\% $(n=475)$ and adenocarcinoma 6.14\% $(n=38)$, with other malignant entries such as other epithelial malignancies, primary tonsillar, mucosaassociated lymphoid tissue or parenchymal lymphomas, connective tissue neoplasias,

Received 05/30/2017

Review began 06/15/2017 Review ended 06/21/2017 Published 07/02/2017

(c) Copyright 2017

Stoyanov et al. This is an open access article distributed under the terms of the Creative Commons Attribution License CC-BY 3.0., which permits unrestricted use, distribution, and reproduction in any medium, provided the original author and source are credited. neuroendocrine and vascular malignancies diagnosed in a minority of cases.

\section{Conclusion}

Considered to be relatively rare, HNC represents a diverse group of oncological entities with individual and specific demographic characteristics. The reported single institution results appear representative of the national incidence and characteristics of HNC.

Categories: Pathology, Oncology, Epidemiology/Public Health

Keywords: head and neck cancer, descriptive analysis, statistics, demographics 


\section{Introduction}

Head and neck cancer (HNC) comprises a diverse group of oncological entities, originating from various tissue types and organ localizations all situated in the topographical region of the head and neck $(H \& N)$ [1-4]. The $H \& N$ region is the sixth most often site for malignancies [5-6]. It is also one of the few medical fields allowing for an uncomplicated endoscopy and diagnostic biopsy through a natural orifice in almost all cases, thus allowing detailed pretreatment diagnostic and staging $[1-4,7-8]$.

The reported worldwide incidence of HNC is approximately $3 \%$ of all cancer cases, with males being affected in nearly $90 \%$ of all cases and epithelial neoplasms representing more than $85 \%$ of all cancer types [2-4, 9-12]. The incidence of HNC in developing countries, however, is higher due to the prevalence of risk factors such as smoking and alcohol consumption. HNC incidence in Bulgaria as per the latest data published by the National Oncological Registry for 2013 reports 1,626 (4.42\%) HNC cases out of 36,825 newly reported cancer cases, for a total population of $7,245,677$ people [13].

The design of this retrospective, single institution study was aimed at establishing the HNC patient demographics and categorizing the individual incidence of $H \& N$ malignancies in a descriptive manner, with regard to their organ of origin and main histopathological type.

\section{Materials And Methods}

All histologically verified cases of HNC from the central pathological archive of a single medical institution - St. Marina University Hospital, Varna, Bulgaria, a tertiary referral center - were reviewed in a retrospective manner. The overall period of data sampling was 47 months (September 2012-July 2016).

The patient identification was based on the unique hospital ID number and Unified Citizen Number. Therefore, a "case" was determined as a patient with HNC, histologically verified in our hospital and not as an individual HNC biopsy.

The study cohort comprised 619 patients, with the total number of biopsy specimens reviewed exceeding 1,500 , as many patients had multiple biopsies in the different stages of their treatment and follow-up.

The establishment of these criteria evaded statistical blurring from patients biopsied or operated more than once in the set timeframe. Recurrent HNC in the set timeframe was also excluded from the statistical analysis. Recurrent HNC however, diagnosed prior to the start of the collection of the data was included as an HNC case. All cases of metastatic disease in the $\mathrm{H} \& \mathrm{~N}$ region were excluded from the statistical analysis, due to the study being aimed only at primary HNC. Locally invasive forms of skin cancer, directly associated with an organ of interest, were also included into the cohort.

Due to the criteria of histological verification of the type of HNC in our institution, patients treated conservatively in our institution for HNC, in wards such as oncology, radiotherapy and palliative care, with a tumor histologically verified in other medical institution, were excluded from the statistical analysis.

\section{Results}

A total of 619 individual HNC cases were registered in the set timeframe across 618 patients. The only patient who had two cases of HNC registered was a 46-year-old female patient first diagnosed with squamous cell carcinoma (SCC), who later developed a mucosa-associated 


\section{Cureus}

lymphoid tissue (MALT) lymphoma in a different $\mathrm{H} \& \mathrm{~N}$ location. These types of synchronous and metachronous tumors are a rare citing worldwide [14]. The yearly distribution of cases, calculated on a patient-yearly basis, was $n=138$ individual cases for 2012, $n=162$ for 2013, $n=$ 153 for 2014, $\mathrm{n}=159$ for 2015 and $\mathrm{n}=170$ for 2016, resulting in an average of 156 unique HNC cases per year (Figure 1A).

Of all the registered HNC cases $76.41 \%(\mathrm{n}=473)$ were diagnosed in males and $23.59 \%(\mathrm{n}=146)$ in females, with male to female ratio of 3.24:1 (Figure 1B). The mean age of diagnosis was 63.84 \pm 12.65 years, median 65 years, with the youngest patient diagnosed being 14 years old and the oldest 103 years of age at the time of diagnosis (Figure $1 C-1 D$ ).

The primary localizations included the larynx 30.37\% $(n=188)$ cases, lips and oral cavity $29.08 \%(n=180)$, pharynx 20.03\% $(n=124)$, salivary glands $10.94 \%(n=68)$, external nose and nasal cavity $4.69 \%(\mathrm{n}=29)$, auricle and external ear canal $2.75 \%(\mathrm{n}=17)$ and sinuses $2.1 \%(\mathrm{n}=$ 13) (Figure 1E).
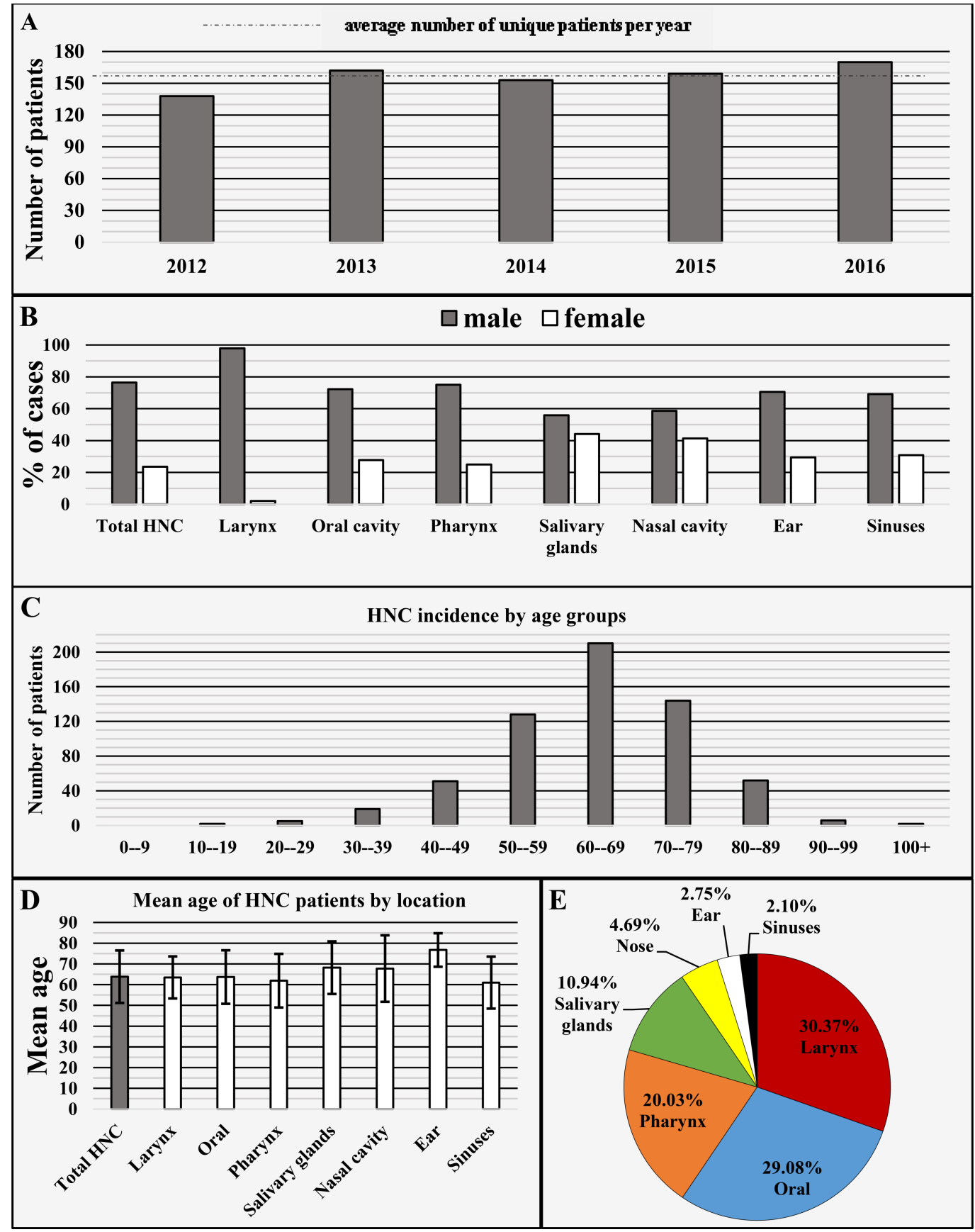


\section{Cureus}

FIGURE 1: Head and neck cancer by distribution of cases on a yearly basis $(A)$, localizations with gender ratio $(B)$, incidence by age group (C), mean age (D) and general distribution by major localizations (E).

HNC: Head and neck cancer.

Regarding the main histopathological groups SCC comprised 76.74\% ( $\mathrm{n}=475)$, adenocarcinoma (AC) $6.14 \%(n=38)$, primary tonsillar, MALT or parenchymal lymphomas $6.14 \%(n=38)$, mucoepidermoid carcinoma $(\mathrm{MeC})$ 4.04\% $(\mathrm{n}=25)$, basocellular carcinoma $(\mathrm{BcC}) 2.26 \%(\mathrm{n}=14)$, different types of sarcoma $1.45 \%(n=9)$, carcinoma ex pleomorphic adenoma (Ca ex PA) $1.29 \%$ $(n=8)$, neuroendocrine carcinoma $(\mathrm{NeC}) 0.97 \%(n=6)$, plasmocytoma $0.65 \%(n=4)$, small round blue cell tumor $0.16 \%(n=1)$ and hemangiopericytoma $0.16 \%(n=1)$ (Figure $2 A)$. The gender ratio of each individual histopathological group is shown in Figure $2 B$.

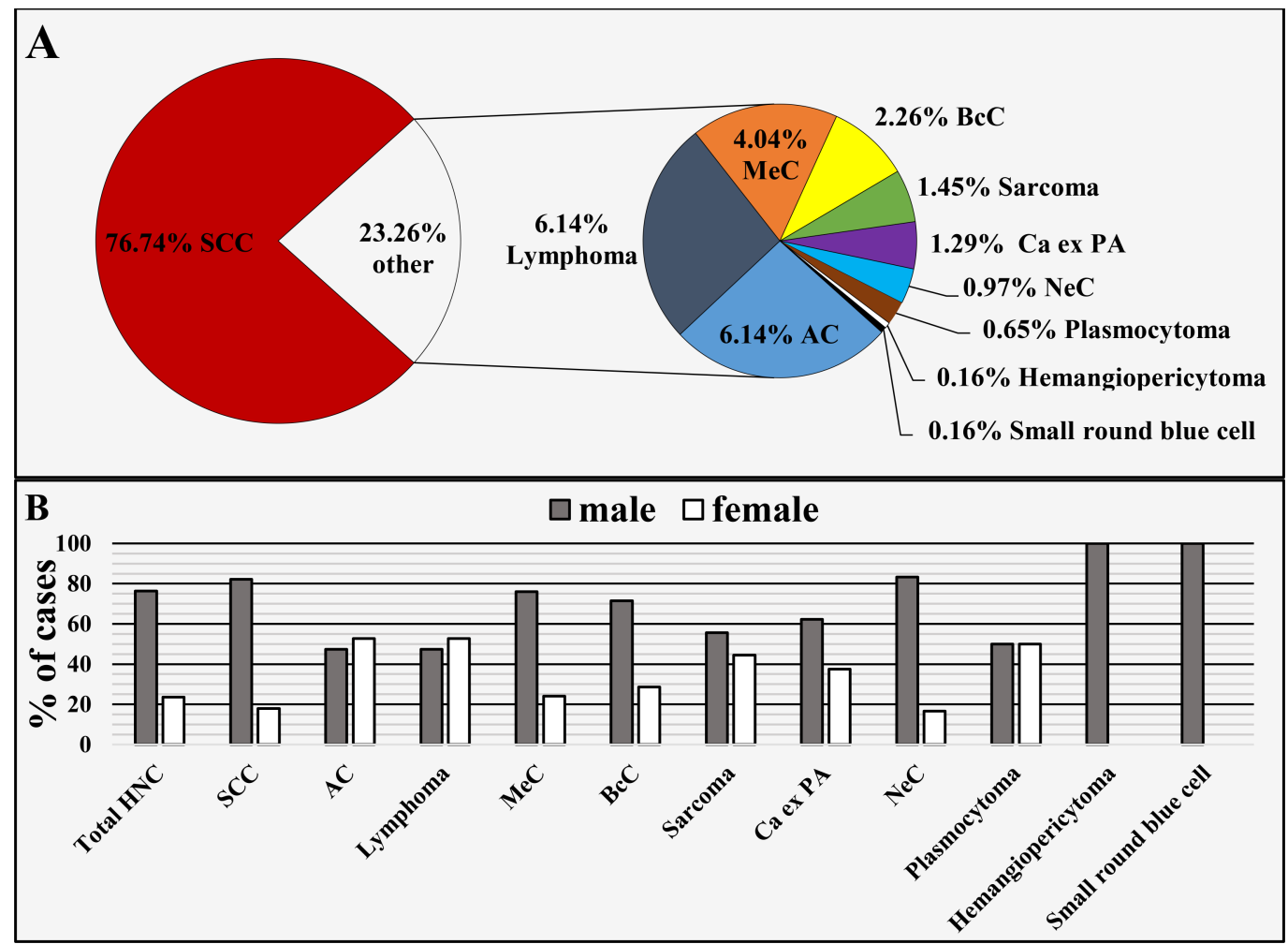

FIGURE 2: Head and neck cancer by main histopathological type (A) and gender ratio of each histopathological type (B).

HNC: Head and neck cancer; SCC: Squamous cell carcinoma; AC: Adenocarcinoma; MeC: Mucoepidermoid carcinoma; BcC: Basocellular carcinoma; Ca ex PA: Carcinoma ex pleomorphic adenoma; NeC: Neuroendocrine carcinoma.

The individual HNC entities by their localization, main histological type, age and gender of the patient and the yearly registered cases with regards to their localizations code in the first revision of the International Classification of Diseases for Oncology 3 (ICD-O 3) are as follows 


\section{Cureus}

[15]:

\section{Larynx and cervical region of the trachea (ICD-O 3: C32.0- C33.9)}

Of all 188 cases registered in this region, $97.88 \%(\mathrm{n}=184)$ were registered in the larynx and $2.12 \%(n=4)$ were registered in the cervical region of the trachea. From all the cases, 92.55\% (n $=174)$ were diagnosed in males and $7.45 \%(n=14)$ in females, with a male to female ratio of 12.43:1 (Figure $1 B$ ), mean age of diagnosis of $63.48 \pm 10.14$ years, median 64 years (Figure $1 D$ and Figure 3A). The different oncological entities were represented with $97.88 \%(n=184)$ SCC, 1.06\% ( $\mathrm{n}=2) \mathrm{MeC}, 0.53 \%(\mathrm{n}=1) \mathrm{AC}$ and $0.53 \%(\mathrm{n}=1)$ hemangiopericytoma (Figure $3 B)$. A total of $6.38 \%(n=12)$ of the cases were registered in the non-invasive stage of carcinoma in situ.
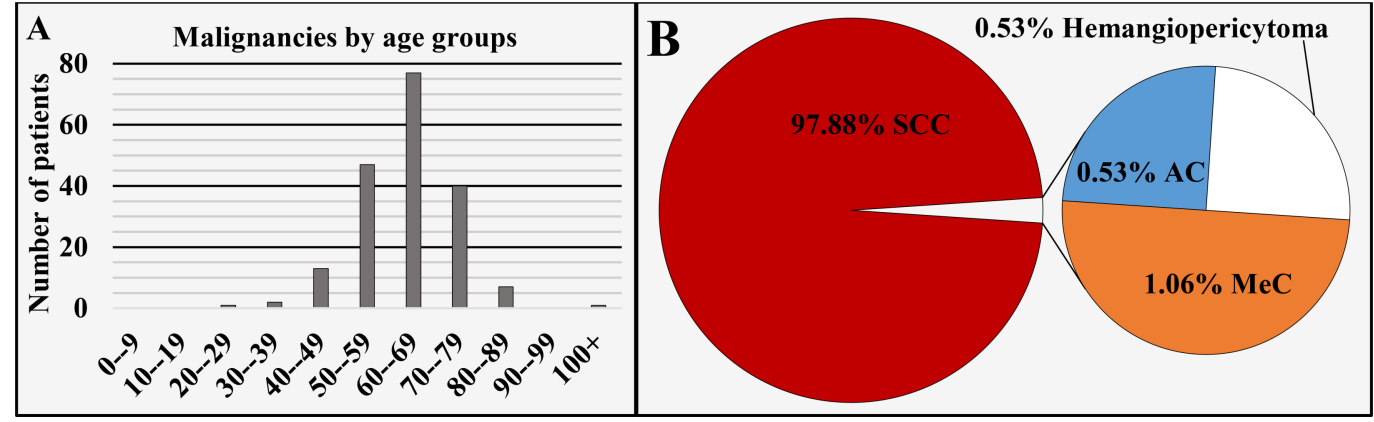

FIGURE 3: Malignancies of the larynx and cervical region of the trachea by age group (A) and main histopathological type (B).

SCC: Squamous cell carcinoma; AC: Adenocarcinoma; MeC: Mucoepidermoid carcinoma.

\section{Lips and oral cavity (ICD-O 3: C00.0-C08.9)}

Of all 180 cases registered in this region, $72.22 \%(n=130)$ were diagnosed in males and 27.78\% $(\mathrm{n}=50)$ in females, with a male to female ratio of 2.6:1 (Figure $1 B$ ), mean age of diagnosis of $63.67 \pm 12.9$ years, median 65 years (Figure $1 D$ and Figure $4 A$ ). The different oncological entities were represented with 79.44\% $(n=143)$ SCC, 7.22\% $(n=13)$ MeC, 5\% $(n=9)$ AC, $2.78 \%(n=5)$ $\mathrm{BcC}, 2.22 \%(\mathrm{n}=4)$ sarcoma, $1.67 \%(\mathrm{n}=3)$ plasmocytoma, 1.11\% $(\mathrm{n}=2)$ lymphoma and 0.56\% ( $\mathrm{n}$ $=1$ ) embryonal undifferentiated small round blue cell tumor (Figure $4 B)$. A total of $2.22 \%(n=4)$ of the cases were registered in the non-invasive stage of carcinoma in situ.
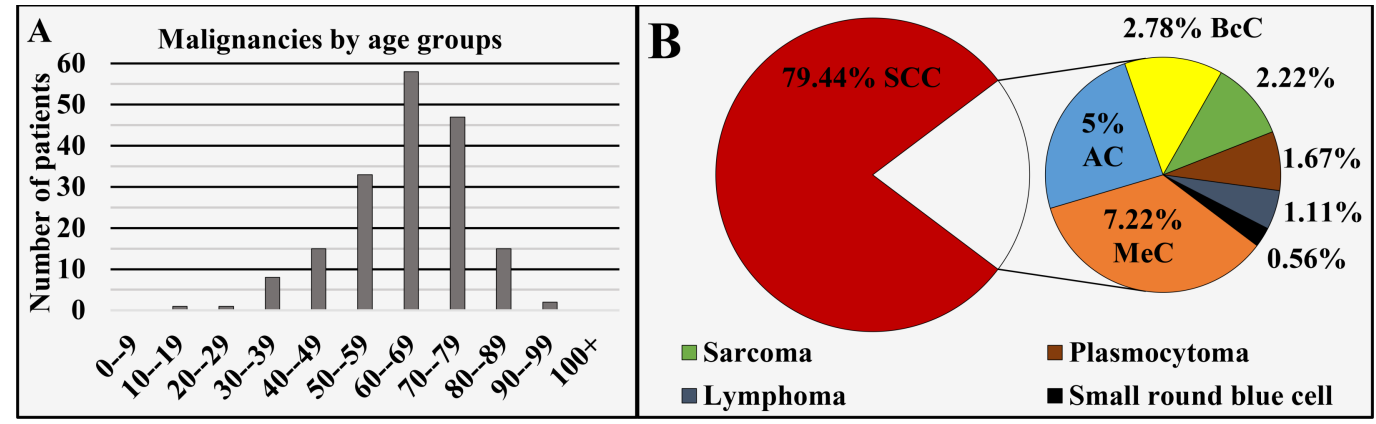

FIGURE 4: Malignancies of the lip and oral cavity by age group (A) and main histopathological type (B). 


\section{Cureus}

SCC: Squamous cell carcinoma; AC: Adenocarcinoma; MeC: Mucoepidermoid carcinoma; BcC: Basocellular carcinoma.

\section{Pharynx (ICD-O 3: C0.9-C14.2)}

Of all 124 cases registered in this region, $75 \%(n=93)$ were diagnosed in males and $25 \%(n=31)$ in females, with a male to female ratio of $3: 1$ (Figure $1 B$ ), mean age of diagnosis of $61.92 \pm$ 12.94 years, median 61 (Figure $1 D$ and Figure $5 \mathrm{~A}$ ). The different oncological entities were represented with 79.03\% $(n=98)$ SCC, 20.16\% $(n=25)$ lymphoma and $0.81 \%(n=1) \mathrm{NeC}$ (Figure $5 B$ ). Malignant formations were isolated from all three portions of the pharynx. A total of $0.81 \%(n=1)$ of the cases were registered in the non-invasive stage of carcinoma in situ.
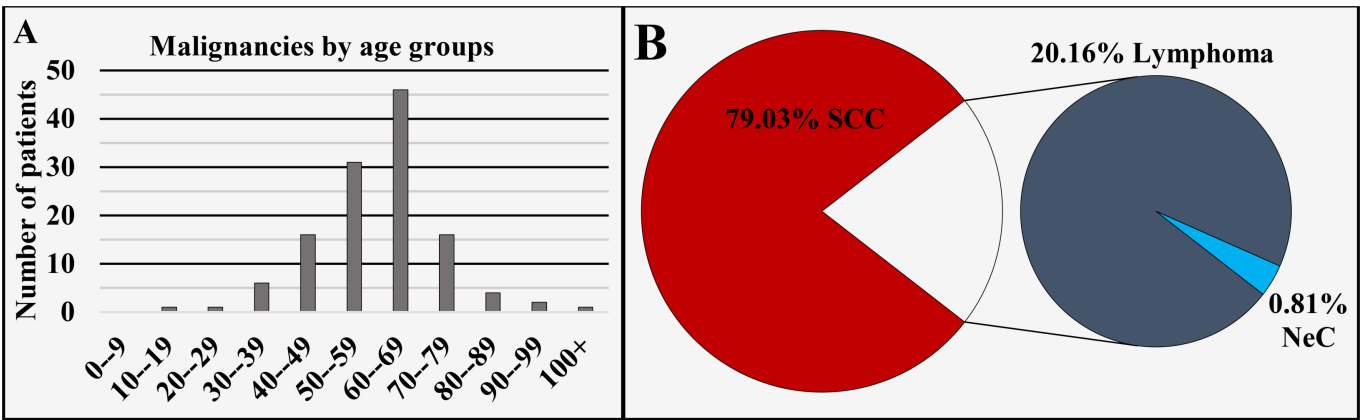

FIGURE 5: Malignancies of the pharynx by age group (A) and main histopathological type (B).

SCC: Squamous cell carcinoma; NeC: Neuroendocrine carcinoma.

\section{Salivary glands (ICD-O 3: C08.0-C08.9)}

Of all 68 cases registered in this region, 55.88\% $(n=38)$ were diagnosed in males and $44.12 \%(n$ $=30$ ) in females, with a male to female ratio of 1.27:1 (Figure $1 B$ ), mean age of diagnosis of $68.19 \pm 12.68$ years, median 70 years (Figure $1 D$ and Figure $6 A$ ). The different oncological entities were represented with $32.36 \%(n=22)$ AC, $23.53 \%(n=16)$ locally invasive SCC, $14.71 \%$ $(\mathrm{n}=10) \mathrm{MeC}, 11.76 \%(\mathrm{n}=8)$ Ca ex PA, 11.76\% $(\mathrm{n}=8)$ lymphoma, 2.94\% $(\mathrm{n}=2)$ NeC, $1.47 \%(\mathrm{n}=$ 1) sarcoma and $1.47 \%(n=1)$ locally invasive $\mathrm{BcC}$ (Figure $6 B)$.
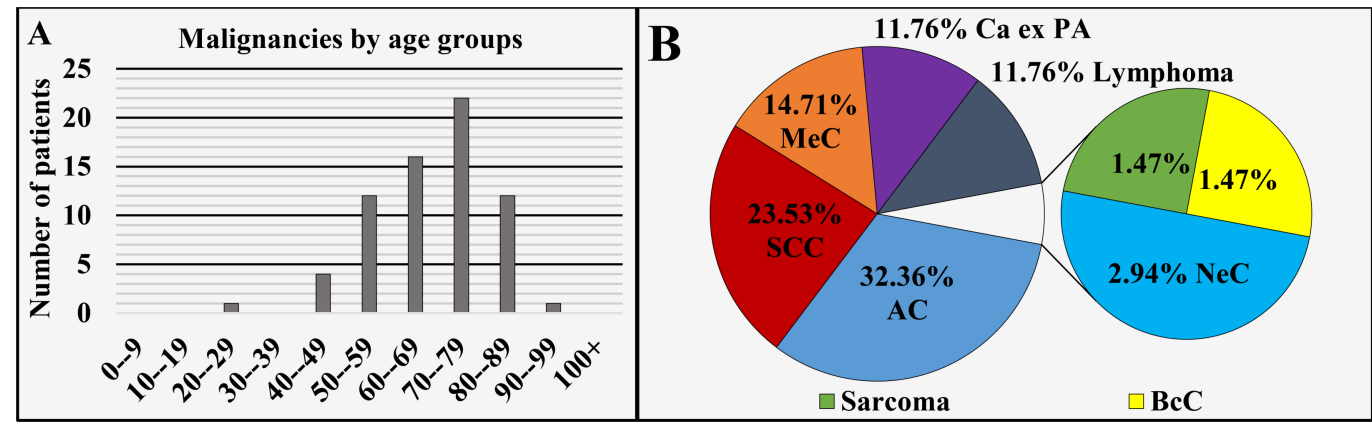

FIGURE 6: Malignancies of the salivary glands by age group (A) and main histopathological type (B).

SCC: Squamous cell carcinoma; AC: Adenocarcinoma; MeC: Mucoepidermoid carcinoma; BcC: 


\section{Cureus}

Basocellular carcinoma; Ca ex PA: Carcinoma ex pleomorphic adenoma; NeC: Neuroendocrine carcinoma.

\section{External nose and nasal cavity (ICD-O 3: C30.0 and C44.3)}

Of all 29 cases registered in this region, 58.61\% $(\mathrm{n}=17)$ were diagnosed in males and 41.39\% (n $=12$ ) in females, with a male to female ratio of 1.42:1 (Figure $1 B$ ), mean age of diagnosis of $67.76 \pm 16.04$ years, median 72 years (Figure $1 D$ and Figure $7 A$ ). The different oncological entities were represented with 58.61\% $(n=17)$ SCC, $17.24 \%(n=5)$ BcC, 6.9\% $(n=2)$ lymphoma, 6.9\% $(\mathrm{n}=2)$ sarcoma, 3.45\% (n=1) NeC, 3.45\% (n=1) AC and 3.45\% (n= 1) plasmocytoma (Figure $7 B$ ).
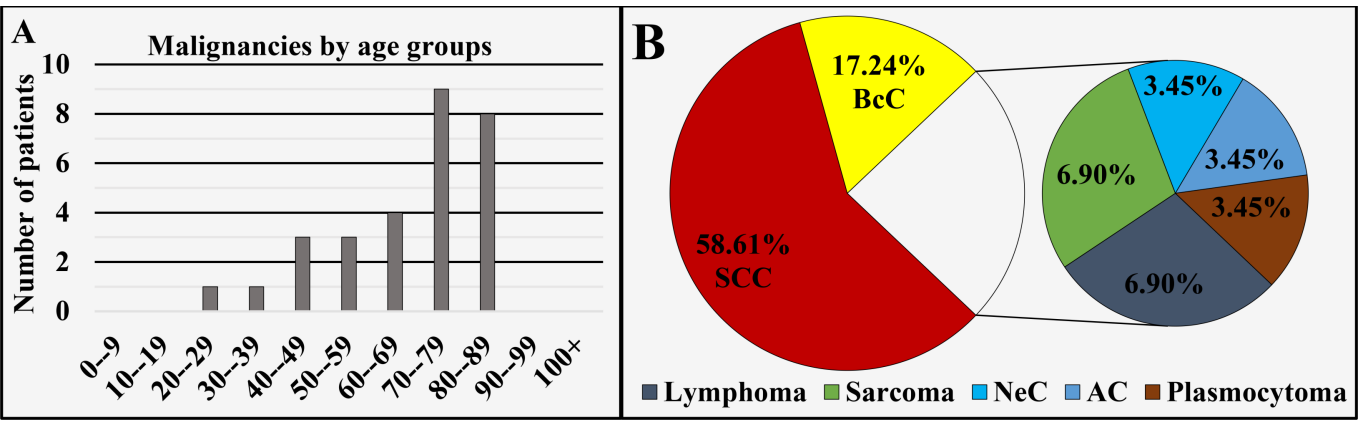

FIGURE 7: Malignancies of the external nose and nasal cavity by age group (A) and main histopathological type (B).

SCC: Squamous cell carcinoma; AC: Adenocarcinoma; BcC: Basocellular carcinoma; NeC: Neuroendocrine carcinoma.

\section{Auricle and external ear canal (ICD-O 3: C44.2 and C44.3)}

Of all 17 cases registered in this region, $70.59 \%(n=12)$ cases were diagnosed in males and $29.41 \%(n=5)$ in females, with a male to female ratio of 2.4:1 (Figure $1 B$ ), mean age of diagnosis of $76.71 \pm 8.08$ years, median 75 years (Figure $1 D$ and Figure $8 A$ ). The different oncological entities were represented with 70.59\% $(n=12)$ SCC, $17.65 \%(n=3)$ BcC and 11.76\% $(\mathrm{n}=2)$ AC (Figure $8 B)$.
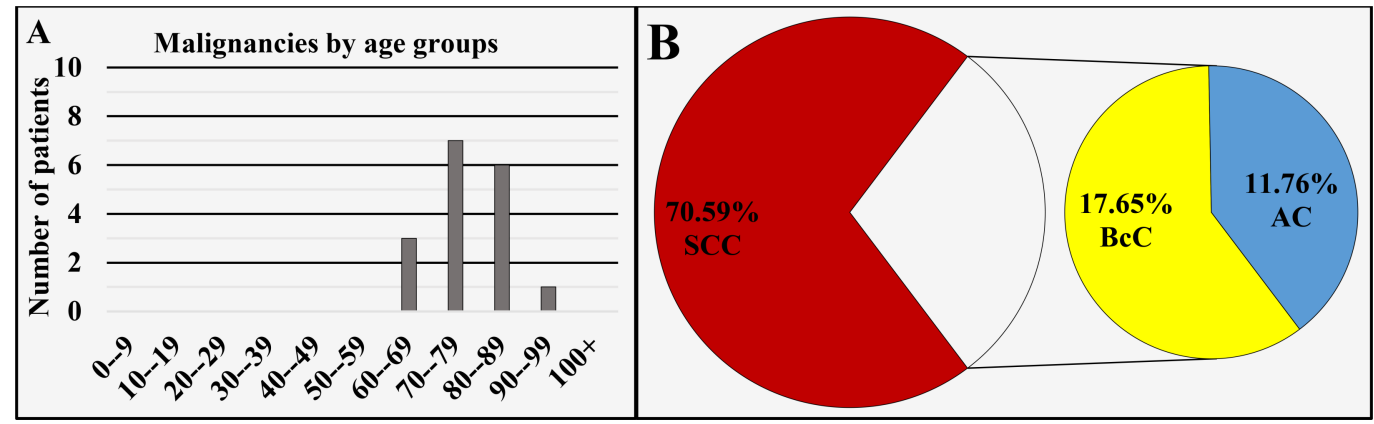

FIGURE 8: Malignancies of the auricle and external ear canal by age group (A) and main histopathological type (B).

SCC: Squamous cell carcinoma; AC: Adenocarcinoma; BcC: Basocellular carcinoma. 


\section{Sinuses (ICD-O 3: C31.0-C31.9)}

Of all 13 cases registered in this region, 69.23\% $(n=9)$ cases were diagnosed in males and $30.77 \%(n=4)$ in females, with a male to female ratio of 2.25:1 (Figure $1 B$ ), mean age of diagnosis of $61 \pm 12.54$ years, median 63 years (Figure $1 D$ and Figure $9 A$ ). The different oncological entities were represented with 38.46\% ( $n=5)$ SCC, $23.09 \%(n=3)$ AC, $15.38 \%(n=2)$ lymphoma, 15.38\% $(\mathrm{n}=2) \mathrm{NeC}$ and $7.69 \%(\mathrm{n}=1)$ sarcoma (Figure $9 B)$.
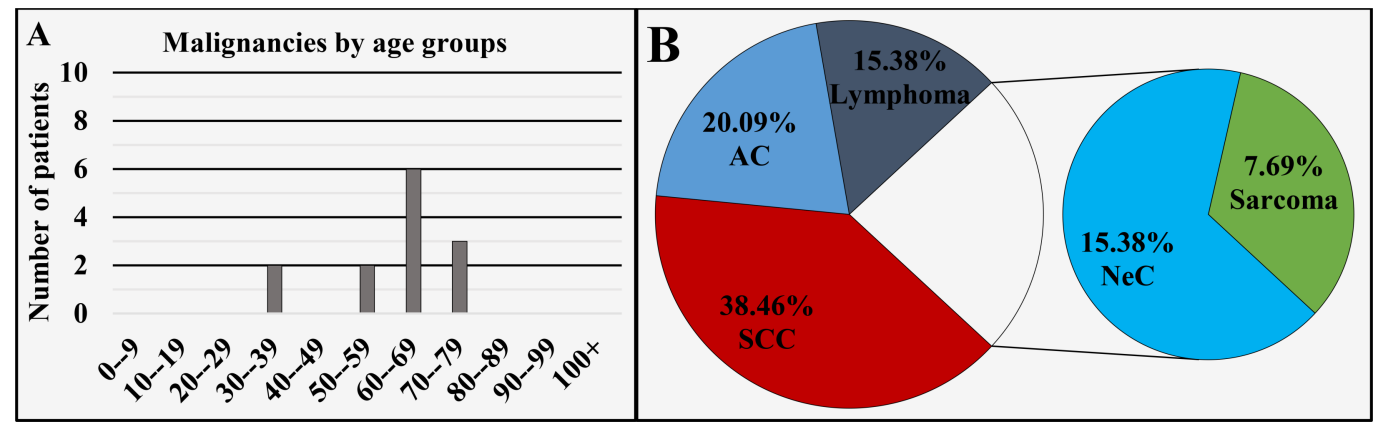

\section{FIGURE 9: Malignancies of the sinuses by age group (A) and main histopathological type (B).}

SCC: Squamous cell carcinoma; AC: Adenocarcinoma; NeC: Neuroendocrine carcinoma.

\section{Discussion}

HNC presents an important medical problem. This anatomical region is the sixth most often localization of malignancies. In up to $90 \%$ of all these cases, the histopathological type reported is SCC $[5-6,16]$. The most pronounced risk factors are tobacco and alcohol consumption [17-19]. SCC, however, is only one of the many histopathological groups of cancers that can arise in the H\&N area. Many other cell types in the region can undergo malignant transformation and give rise to malignant growth, requiring different clinicopathological criteria for grading and staging, with different treatment strategies and prognosis for each different tumor type.

In our report, exclusions from the list of presented data are entities from some organs situated in the $H \& N$ area, the malignant tumors, which fall into a different category, due to their organ specifics and characteristics. All cases of metastatic HNC were excluded from the study cohorts, as we aimed only at establishing the incidence and demographics only of primary cases. Other omitted entities include thyroid and parathyroid malignancies, which fall into the category of endocrine neoplasms. Lymph nodes, which fall into the category of hematological malignancies, if not metastatic and skin malignancies, not directly associated with an organ of interest, which fall into the category of skin cancer. More specific cancer types found in the $\mathrm{H} \& \mathrm{~N}$ region were also excluded, such as odontological and bone malignancies, which fall into separate groups of their own and central nervous system and ocular malignancies, falling into the categories of central nervous system neoplasms and ocular malignancies, respectively. Despite this, some statistical analysis includes these tumors, based purely on their topographical localizations [3, 20].

We observed some variation in the incidence of HNC by localization of the primary malignancy on yearly basis, which we would attribute to non-medical reasons, such as the natural shift in 
the referral preferences of the primary and secondary centers, the preferences of the patients, as well as changes in the capacities of related departments in our institution. Despite all this, the total incidence of HNC remains near unchanged in the separate years and the mean age of diagnosis is nearly identical across most localizations, as reported by other working groups [21]. However, the retrospective nature of the study and its short timeframe, combined with it being carried only in one medical center make it hard to establish a tendency for dynamic in HNC demographics, location and main histopathological site, which could be noted on a more general scale as in nationwide surveys [13].

The reported figures for gender ratio between localizations show a lower male to female ratio than the worldwide data, but higher than the figures reported in the developed countries. We observed a lower percentage of SCC than that of the global population [2-4, 21].

The frequencies observed at our institution correspond to the overall proportions for the different primary localizations and histological types as compared to the latest published national oncology reports, where the HNC rates follow a nearly identical rate and statistical curves as the currently reported values, for the respective years [13, 22-24].

With the widespread prevalence of the major known risk factors for developing HNC across the Bulgarian population, the results seem to be representative and are a critical reflection of the level of medical care in the country and further need to increase the quality and volume of activities in prevention politics [19, 25-28].

Our retrospective analysis does not include some casuistically rare types of HNC, which can be registered in the $\mathrm{H} \& \mathrm{~N}$ region, due to their extreme rarity and lack of patients with such diagnoses in the period of data sampling. These rare tumor types are very rarely included in any statistical analysis and are most often just reported as individual cases [2-4, 29].

Some HNC patients may have a secondary malignancy in another anatomical region or a metastatic HNC formation, however, our study design was aimed only at the H\&N region and primary HNC. Only one patient with a second type of HNC in the set timeframe was reported. Our study design appears quite insensitive to such data and this single case observed is not representative for synchronous malignancies. Anyhow, data on synchronous and metachronous HNC is rarely reported [14].

\section{Conclusions}

HNC is a relatively rare subset of cancer in Bulgaria, accounting for just above $4 \%$ of cancer cases. It, however, represents a major and likely increasing medical and social problem. Our data offers valuable statistical insight for the demographics of HNC patient in Bulgaria, while comparing the localizations of interest, main histopathological groups and the rate of yearly diagnosed cases, for the first time in such depth. Future public health efforts at primary prevention through reducing the modifiable risk factors should be encouraged.

\section{Additional Information \\ Disclosures}

Human subjects: Consent was obtained by all participants in this study. Informed consent obtained. Animal subjects: All authors have confirmed that this study did not involve animal subjects or tissue. Conflicts of interest: In compliance with the ICMJE uniform disclosure form, all authors declare the following: Payment/services info: All authors have declared that no financial support was received from any organization for the submitted work. Financial relationships: All authors have declared that they have no financial relationships at present or 
within the previous three years with any organizations that might have an interest in the submitted work. Other relationships: All authors have declared that there are no other relationships or activities that could appear to have influenced the submitted work.

\section{Acknowledgements}

The authors would like to thank all of the medical and non-medical personnel, past and present, of the "St. Marina" University Hospital - Varna, Bulgaria, who while performing their medical and humanitarian duties allowed for the realization of this study.

\section{References}

1. Argiris A, Karamouzis MV, Raben D, et al.: Head and neck cancer. Lancet. 2008, 371:16951709. 10.1016/S0140-6736(08)60728-X

2. Sturgis EM, Wei Q, Spitz MR: Descriptive epidemiology and risk factors for head and neck cancer. Semin Oncol. 2004, 31:726-733. 10.1053/j.seminoncol.2004.09.013

3. Davies L, Welch HG: Epidemiology of head and neck cancer in the United States . Otolaryngol Head Neck Surg. 2006, 135:451-457. 10.1016/j.otohns.2006.01.029

4. Mehanna H, Paleri V, West CM, et al.: Head and neck cancer - part 1: epidemiology, presentation, and prevention. BMJ. 2010, 341:c4684. 10.1136/bmj.c4684

5. Black RJ, Bray F, Ferlay J, et al.: Cancer incidence and mortality in the European Union: cancer registry data and estimates of national incidence for 1990. Eur J Cancer. 1997, 33:1075-1107. 10.1016/S0959-8049(96)00492-3

6. Hoffman HT, Karnell LH, Funk GF, et al.: The National Cancer Data Base report on cancer of the head and neck. Arch Otolaryngol Head Neck Surg. 1998, 124:951-962. 10.1001/archotol.124.9.951

7. Ang KK, Trotti A, Brown BW, et al.: Randomized trial addressing risk features and time factors of surgery plus radiotherapy in advanced head-and-neck cancer. Int J Radiat Oncol Biol Phys. 2001, 51:571-578. 10.1016/S0360-3016(01)01690-X

8. Lavertu P, Bonafede JP, Adelstein DJ, et al.: Comparison of surgical complications after organpreservation therapy in patients with stage III or IV squamous cell head and neck cancer. Arch Otolaryngol Head Neck Surg. 1998, 124:401-406. 10.1001/archotol.124.4.401

9. Sturgis EM, Cinciripini PM: Trends in head and neck cancer incidence in relation to smoking prevalence: an emerging epidemic of human papillomavirus-associated cancers?. Cancer. 2007, 110:1429-1435. 10.1002/cncr.22963

10. Schantz SP, Yu GP: Head and neck cancer incidence trends in young Americans, 1973-1997, with a special analysis for tongue cancer. Arch Otolaryngol Head Neck Surg. 2002, 128:268274. 10.1001/archotol.128.3.268

11. Marur S, Forastiere AA: Head and neck cancer: changing epidemiology, diagnosis, and treatment. Mayo Clin Proc. 2008, 83:489-501. 10.4065/83.4.489

12. Jemal A, Siegel R, Ward E, et al.: Cancer statistics, 2006. CA Cancer J Clin. 2006, 56:106-130. 10.3322/canjclin.56.2.106

13. Bulgarian National Cancer Registry: Cancer incidence in Bulgaria, 2013. Valerianova Z, Dimitrova N, Petkova Y, et al. (ed): Paradigma, Sofia; 2015.

14. Schwartz LH, Ozsahin M, Zhang GN, et al.: Synchronous and metachronous head and neck carcinomas. Cancer. 1994, 74:1933-1938. 10.1002/1097-0142(19941001)74:7<1933::AIDCNCR2820740718>3.0.CO;2-X

15. World Health Organisation: International classification of diseases for oncology (3 ed.) first revision. Fritz A, Percy C, Jack A, et al. (ed): World Health Organisation, Malta; 2013.

16. Levi F, Lucchini F, La Vecchia C, et al.: Trends in mortality from cancer in the European Union, 1955-94. Lancet. 1999, 354:742-743. 10.1016/S0140-6736(99)01909-1

17. Lewin F, Norell SE, Johansson H, et al.: Smoking tobacco, oral snuff, and alcohol in the etiology of squamous cell carcinoma of the head and neck: a population-based case-referent study in Sweden. Cancer. 1998, 82:1367-1375. 10.1002/(SICI)1097-

0142(19980401)82:7<1367::AID-CNCR21>3.0.CO;2-3

18. Reichart PA: Identification of risk groups for oral precancer and cancer and preventive measures. Clin Oral Investig. 2001, 5:207-213. 10.1007/s00784-001-0132-5 
19. Georgiev G, Nikiforova L, Iliev P, et al.: Tobacco exposure in laryngeal cancer patients [Article in Bulgarian]. MedInfo. 2015, 12:52-55.

20. AAssar OS, Fischbein NJ, Caputo GR, et al.: Metastatic head and neck cancer: role and usefulness of FDG PET in locating occult primary tumors. Radiology. 1999, 210:177-181. 10.1148/radiology.210.1.r99ja48177

21. Simard EP, Torre LA, Jemal A: International trends in head and neck cancer incidence rates: differences by country, sex and anatomic site. Oral Oncol. 2014, 50:387-403.

10.1016/j.oraloncology.2014.01.016

22. Bellomo R, Warrillow SJ, Reade MC: Why we should be wary of single-center trials . Crit Care Med. 2009, 37:3114-3119. 10.1097/CCM.0b013e3181bc7bd5

23. Song JW, Chung KC: Observational studies: cohort and case-control studies. Plast Reconstr Surg. 2010, 126:2234-2242. 10.1097/PRS.0b013e3181f44abc

24. Bulgarian National Cancer Registry: Cancer incidence in Bulgaria, 2012. Valerianova Z, Dimitrova N, Tonev S, et al. (ed): Paradigma, Sofia; 2014.

25. Slots J, Saygun I, Sabeti M, et al.: Epstein-Barr virus in oral diseases. J Periodontal Res. 2006, 41:235-244. 10.1111/j.1600-0765.2006.00865.x

26. Bray F, Lortet-Tieulent J, Znaor A, et al.: Patterns and trends in human papillomavirus-related diseases in Central and Eastern Europe and Central Asia. Vaccine. 2013, 31:32-45. 10.1016/j.vaccine.2013.02.071

27. Balabanova D, McKee M: Patterns of alcohol consumption in Bulgaria. Alcohol Alcohol. 1999, 34:622-628. 10.1093/alcalc/34.4.622

28. Balabanova D, Bobak M, McKee M: Patterns of smoking in Bulgaria . Tob Control. 1998, 7:383385.

29. Lapierre A, Selmaji I, Samlali H, et al.: Esthesioneuroblastoma: a single institution's experience and general literature review. Cancer Radiother. 2016, 20:783-789.

10.1016/j.canrad.2016.05.015 